\title{
Statistical Mechanics of Quantum Lattice Systems without Translation Invariance
}

\author{
H. Roos \\ Institut für Theoretische Physik, Universität Göttingen, Göttingen, Federal Republic of Germany
}

Received December 10, 1974

\begin{abstract}
The well-known results concerning the equilibrium of a translation invariant quantum lattice system - existence of the pressure and of the time automorphisms, variational principle for the pressure - are generalized to a large class of quantum lattice systems with potentials not exhibiting covariance under the group of lattice translations.
\end{abstract}

\section{Introduction}

It is well known [1] that for a quantum lattice system with a suitable interaction $\phi(X), X \subset \mathbb{Z}^{v}$, the pressure $P(\phi)$ and the group of time automorphisms $\tau_{t}(\phi)$ exist in the thermodynamic limit. In proving these results one makes repeatedly use of the translation covariance of the interaction:

$$
\phi(X+a)=\tau_{a} \phi(X)
$$

where $X+a, a \in \mathbb{Z}^{v}$, denotes the set obtained by translation of $X$, and $\tau_{a}$ is the canonical isomorphism of the bounded operators on $\mathscr{H}_{X}$ onto the bounded operators on $\mathscr{H}_{X+a}, \tau_{a}: B\left(\mathscr{H}_{X}\right) \rightarrow B\left(\mathscr{H}_{X+a}\right)$. (Our notation is as usual: to each site $x$ of the $v$-dimensional lattice $\mathbb{Z}^{v}$ we attribute a copy $\mathscr{H}_{x}$ of a finite dimensional Hilbert space $\mathscr{H}$, and $\left.\mathscr{H}_{X}=\bigotimes_{x \in X} \mathscr{H}_{x} \cdot\right)$ But there are systems for which (1) does not hold: consider, for instance, two-component crystals, crystals with impurities, or systems with inhomogeneous external fields, or even stationary non-equilibrium systems. We shall concentrate here on systems in equilibrium. It will turn out that, due to the fact that our observable algebra is assumed to be quasi-local, $\tau_{t}(\phi)$ can be defined for all interactions satisfying a temperedness condition which is an obvious generalization of the usual one: There is a norm $\|\phi\|_{f}, f(\xi)=e^{\alpha \xi}$, which has to be finite; if $\phi$ satisfies (1), $\|\phi \mid\|_{f}$ conincides with the usual norm $\|\phi\|_{f}$ which is assumed to be finite in [2], where the existence of $\tau_{t}(\phi)$ is demonstrated for translation covariant potentials. Furthermore, one can show that $\tau_{t}(\phi)$ depends continuously on $\phi$. These results are contained in Section III. (Precise definitions and notations will be found in the following section.) The existence of the pressure is ensured by a weak form of temperedness, but, in addition, Eq. (1) has to be replaced by a condition which guarantees the existence of a mean of the "local" pressures $P_{\Lambda_{0}+x}(\phi)$ for fixed $\Lambda_{0}$. We can deal either with "asymptotically translation covariant potentials" describing a lattice with a locally disturbed potential, or, what is more interesting, treat potentials describing "randomly scattered impurities" in addition to the regular lattice interaction. This class of potentials will turn out to be a fairly large one. This is done in Sections IV-VI. 
Finally, in the last section, a general variational principle will be proved:

$$
P(\phi)=\sup _{\varrho \in \mathscr{S}}(\widetilde{s}(\varrho)-E(\varrho ; \phi)),
$$

where $\bar{s}(\varrho)$ is the mean entropy density and $E(\varrho ; \phi)$ the energy density in the state $\varrho$ which is not necessarily translation invariant.

\section{Notations and Definitions}

Our notations are mainly those of [1]. By $X, Y Z, \Lambda, \Delta, \ldots$ we always denote finite subsets of the lattice $\mathbb{Z}^{v} ; N(X)$ is the number of sites in $X . X+a, a \in \mathbb{Z}^{v}$ denotes the set $X$ translated by $a$.

An interaction $\phi$ is a function on the lattice $\mathbb{Z}^{v}: X \mapsto \phi(X) \in B\left(\mathscr{H}_{X}\right), \phi$ selfadjoint; we identify $\mathscr{H}_{X \cup Y}$ with $\mathscr{H}_{X} \otimes \mathscr{H}_{Y}$ and, similarly, $A \in B\left(\mathscr{H}_{X}\right)$ with $A \otimes \mathbb{1}_{Y}$ $\in B\left(\mathscr{H}_{X \cup Y}\right)$ if $X \cap Y=\emptyset$. With this identification, the algebra $\mathscr{A}$ of observables is the norm closure of the inductive limit $\Lambda \rightarrow \infty$ of the local algebras $\mathscr{A}_{\Lambda} \simeq B\left(\mathscr{H}_{A}\right)$. An element $A \in \mathscr{A}$ is called strictly local if $A \in \mathscr{A}_{A}$ for some $\Lambda$.

The Hamiltonians associated with $\phi$ are $H_{\Lambda}(\phi)=\sum_{X \subset A} \phi(X)$. Given $H_{\Lambda}$, we define the local pressures and the local time automorphismus

$$
\begin{aligned}
P_{\Lambda}(\phi) & =N(\Lambda)^{-1} \log \operatorname{Tr}_{\Lambda} \exp \left(-H_{\Lambda}(\phi)\right), \quad \operatorname{Tr}_{\Lambda}=\text { trace over } \mathscr{H}_{\Lambda}, \\
\tau_{t}^{\Lambda}(\phi) A & =\exp \left(\text { it } H_{\Lambda}(\phi)\right) A \exp \left(-\mathrm{it} H_{\Lambda}(\phi)\right), \quad A \subset \mathscr{A}_{\Lambda_{1}}, \quad \Lambda_{1} \subset A ;
\end{aligned}
$$

and the pressure and the time automorphism group

$$
P(\phi)=\lim _{\Lambda \rightarrow \infty} P_{\Lambda}(\phi), \quad \tau_{t}(\phi)=\text { extension to } \mathscr{A} \text { of } \lim _{\Lambda \rightarrow \infty} \tau_{t}^{\Lambda}(\phi),
$$

provided the limits exist.

We denote the van Hove limit $\Lambda \rightarrow \infty$ by $\lim _{A_{\vec{A}}^{\infty}}$. Likewise, $\lim _{A_{\vec{R}}^{\infty}}$ means the limit in the sense of Robinson: $\Lambda \rightarrow \infty$ such that $\Lambda$ eventually contains every finite subset of $\mathbb{Z}^{v}$. For translationally non-covariant potentials, it is necessary to take $\lim _{\Lambda \overrightarrow{H, R} \infty}$ in defining $P(\phi)$; the time automorphism $\tau_{t}(\phi)$ are obtained with the help of the Robinson limit.

$\mathscr{S}=\left\{\varrho, \varrho^{\prime}, \ldots\right\}$ denotes the set of states over the algebra $\mathscr{A}$; the subset of translation invariant states is denotes by $\mathscr{S}_{0}$. The restrictions of a state $\varrho$ to local algebras $\mathscr{A}_{A}$ are given by positive trace class operators of trace 1 which will be denoted by $\varrho_{A}: \varrho(A)=\operatorname{Tr}_{\Lambda} \varrho_{\Lambda} A$ for $A \in \mathscr{A}_{\Lambda}$. Notice that, for $\Lambda \subset \Lambda^{\prime}, \varrho_{\Lambda}=\operatorname{Tr}_{\Lambda^{\prime} \backslash \Lambda} \varrho_{\Lambda^{\prime}}$ (= partial trace over $\mathscr{H}_{\Lambda^{\prime} \backslash \Lambda}$ ). The local entropy associated with a state $\varrho$ is given by

$$
S_{\varrho}(\Lambda)=-\operatorname{Tr}_{\Lambda} \varrho_{\Lambda} \log \varrho_{\Lambda} .
$$

Let $f(\xi)$ be a positive function over $\mathbb{R}^{+}$; we define two $f$-norms of $\phi$ by

$$
\begin{gathered}
\|\phi\|_{f}=\sup _{x \in \mathbb{Z}^{v}}\|\phi\|_{f}^{x}, \quad\|\phi\|_{f}^{x}=\sum_{X \ni x}\|\phi(X)\| f(N(X)) ; \\
\|\phi\|_{f}=\sum_{k=1}^{\infty} f(k) \sup _{x \in \mathbb{Z}^{v}} \sum_{X: X \ni x, N(X)=k}\|\phi(X)\| .
\end{gathered}
$$


Clearly, $\|\phi\|_{f} \leqq\|\phi\|_{f}$, both norms coincide with the usual one for translation covariant interactions. By $\overline{\mathscr{B}}_{f}, \hat{\mathscr{B}}_{f}, \mathscr{B}_{f}^{T}$ we denote the following Banach spaces of interactions:

$$
\begin{aligned}
\mathscr{\mathscr { B }}_{f} & =\left\{\phi,\|\phi\|_{f}<\infty\right\}, \\
\hat{\mathscr{B}}_{f} & =\left\{\phi ;\|\phi\|_{f}<\infty\right\}, \\
\mathscr{B}_{f}^{T} & =\left\{\phi \in \overline{\mathscr{B}}_{f} ; \phi \text { translation covariant }\right\} .
\end{aligned}
$$

The time automorphisms $\tau_{t}(\phi)$ can be defined for all $\phi \in \hat{\mathscr{B}}_{f}$ with $f(\xi) \geqq e^{\alpha \xi}$ for some $\alpha>0$; the pressure will be shown to exist for $\phi$ from a subspace of $\overline{\mathscr{B}}_{1 / \xi}$. For that purpose, we have to find a substitute for the translation covariance of $\phi$. Equation (1) can be replaced by the condition of asymptotic translation covariance (ATC):

Definition 2.1. An interaction $\phi$ is called $f$-asymptotically translation covariant, $\phi \in \mathscr{B}_{f}^{a}$, if

(i) $\phi \in \overline{\mathscr{B}}_{f}$,

(ii) for arbitrary $\varepsilon>0$, there exists a finite set $\Delta_{\varepsilon}(\phi) \subset \mathbb{Z}^{v}$ such that

$$
\sum_{X \ni x}\left\|\phi(X+a)-\tau_{a} \phi(X)\right\| f(N(X))<\varepsilon
$$

for all $x$ and $a$ for which $x \notin \Delta_{\varepsilon}(\phi), x+a \notin \Delta_{\varepsilon}(\phi)$.

Besides ATC, this condition means a sort of uniform temperedness which we need in order to show that any $\phi \in \mathscr{B}_{f}^{a}$ can be approximated by finite range potentials. Such a potential could describe a crystal with a local impurity. A more interesting case is the following: we consider a potential $\phi=\phi_{T}+\phi_{R}$ with a translation covariant part $\phi_{T}$ and a part $\phi_{R}$ describing randomly scattered impurities (RSI-potential).

Definition 2.2. A RSI-potential $\phi_{R}=\sum_{x \in I} \phi^{x}+\phi_{R}^{W}$ is given by

(i) a set $I \subset \mathbb{Z}^{v}$ of "randomly distributed" impurity sites, i.e. obeying condition $(R)$ to be defined in Section V,

(ii) potentials $\phi^{x}$ "centered around $x$ ", i.e.

$$
\phi^{x}(X)=0 \quad \text { unless } \quad x \in X,
$$

which describe the interaction of the impurity at $x$ with the undisturbed lattice; furthermore,

$$
\phi^{x}(X)=\tau_{x-y} \phi^{y}(X-(x-y)), \quad \text { if } \quad x, y \in I,
$$

(iii) a potential $\phi_{R}^{W}$ describing the interaction among the impurities,

$$
\begin{gathered}
\phi_{R}^{W}(X)=0 \text { unless } \quad X \subset I, \\
\tau_{a} \phi_{R}^{W}(X)=\phi_{R}^{W}(X+a) \text { if } X \subset I, \quad X+a \subset I .
\end{gathered}
$$

Equations (8) and (9) suggest that $\phi_{R}^{W}$ can be derived from a translation covariant potential, say, $\phi^{W}$ by

$$
\phi_{R}^{W}(X)=\left\{\begin{array}{cl}
\phi^{W}(X) & \text { if } X \subset I, \\
0 & \text { otherwise, }
\end{array} \quad \tau_{a} \phi^{W}(X)=\phi^{W}(X+a) .\right.
$$


In view of physical applications, this seems reasonable. Equations (7)-(9) imply that we are dealing with one sort of impurities only. Actually, we can cope with an appreciably larger class of "generalized RSI-potential" (see Definition 5.4) containing sums of RSI-potentials and potentials describing interactions among different kinds of impurities.

The definitions of $\bar{s}(\varrho)$ and $E(\varrho ; \phi)$ will be given in the last section.

\section{Existence of Time Automorphisms}

In this section, we consider interactions $\phi \in \hat{\mathscr{B}}_{f}$, with $f(\xi) \geqq e^{\alpha \xi}$ for some positive $\alpha$. We put $\alpha=1$ in order to simplify the formulas.

Definition 3.1. The $\Lambda-f$-seminorm of $\phi$ is defined by

$$
\|\phi\|\left\|_{f}^{\Lambda}=\sum_{k=1}^{N(\Lambda)} f(k) \sup _{x \in \Lambda} \sum_{X: x \in X^{\prime}} \sum_{C A, N(X)=k}\right\| \phi(X) \| .
$$

Clearly, $\|\phi\|_{f}^{\Lambda}$ increases with increasing $\Lambda$, and $\sup _{\Lambda \subset \mathbb{Z}^{v}}\|\phi\|_{f}^{\Lambda}=\|\| \phi \|_{f}$. The results of this section depend on the following.

Lemma 3.2. Assume $\phi_{1}, \ldots, \phi_{n} \in \hat{\mathscr{B}}_{f}, f(\xi) \geqq e^{\xi-1}$, and let $A$ be strictly local, say, $A \in \mathscr{A}_{\Lambda_{0}}$. Then, for $\Lambda_{i} \supset \Lambda_{0}, i=1,2, \ldots, n$,

$$
\begin{aligned}
& \left\|\left[H_{\Lambda_{n}}\left(\phi_{n}\right),\left[H_{\Lambda_{n-1}}\left(\phi_{n-1}\right),\left[\ldots\left[H_{\Lambda_{1}}\left(\phi_{1}\right), A\right] \ldots\right]\right]\right]\right\| \\
& \quad=\left\|\sum_{X_{1} \subset \Lambda_{1}} \cdots \sum_{X_{n} \subset A_{n}}\left[\phi_{n}\left(X_{n}\right),\left[\phi_{n-1}\left(X_{n-1}\right),\left[\ldots\left[\phi_{1}\left(X_{1}\right), A\right] \ldots\right]\right]\right]\right\| \\
& \quad \leqq n ! 2^{n}\|A\| \exp \left(N\left(\Lambda_{0}\right)\right) \prod_{i=1}^{n}\left\|\phi_{i}\right\|_{f}^{A_{i}} .
\end{aligned}
$$

This is a generalization of Lemma 7.6.1 of [1], and can be proved by the same method.

Theorem 3.3. Assume $\phi \in \hat{\mathscr{B}}_{f}$ with $f(\xi) \geqq e^{\xi-1}$. Then, for $A \in \mathscr{A}_{\Lambda_{0}}$,

$$
\tau_{t}(\phi) A=\lim _{\substack{A \rightarrow \infty \\ R}} \tau_{t}^{\Lambda}(\phi) A=\lim _{\substack{A \rightarrow \infty \\ R}} \exp \left(\text { it } H_{\Lambda}(\phi)\right) A \exp \left(-i t H_{\Lambda}(\phi)\right)
$$

exists, defining a strongly continuous one-parameter group of automorphisms $\tau_{t}: \mathbb{R} \rightarrow$ Aut $\mathscr{A}$.

Proof. Put $\phi_{i}=\phi$ and $\Lambda_{i}=\Lambda, i=1, \ldots, n$, in Lemma 3.2, then

$$
\left\|\left[H_{\Lambda}(\phi), A\right]^{(n)}\right\| \leqq n !\|A\| \exp \left(N\left(\Lambda_{0}\right)\right)\left(2\|\phi\|_{f}\right)^{n},
$$

where $[\ldots]^{(n)}$ denotes the iterated commutator:

$$
[B, A]^{(0)}=A, \quad[B, A]^{(n)}=\left[B,[B, A]^{(n-1)}\right] .
$$

We have

$$
\tau_{t}^{\Lambda}(\phi) A=\sum_{n=0}^{\infty} \frac{(i t)^{n}}{n !}\left[H_{\Lambda}(\phi), A\right]^{(n)}
$$

The rest of the proof can be copied word by word from the proof of Theorem 7.6.2 of [1] which rests on (12) and the analog of (11). 
Remark 3.4. Contrary to what holds in the translation invariant case, the lattice translations do no longer commute with the time automorphisms defined by an arbitrary $\phi \in \hat{\mathscr{B}}_{f}$. We have instead

where $\phi_{x}$ is defined by

$$
\tau_{t}(\phi) \tau_{x}=\tau_{x} \tau_{t}\left(\phi_{x}\right)
$$

$$
\phi_{x}(X)=\tau_{-x} \phi(X+x) .
$$

Theorem 3.5. For fixed $A \in \mathscr{A}$ and fixed $t$, the mapping $\phi \mapsto \tau_{i}(\phi) A$ is normcontinuous on $\hat{\mathscr{B}}_{f}$ equipped with its Banach space topology.

Proof. We consider a sequence $\phi_{\alpha}$ converging in $\|\ldots\|_{f}$-norm to $\phi$. Notice that

$$
\begin{aligned}
& {\left[H_{\Lambda}\left(\phi_{\alpha}\right), A\right]^{(n)}-\left[H_{\Lambda}(\phi), A\right]^{(n)}} \\
& \quad=\sum_{r=0}^{n-1}\left[H_{\Lambda}\left(\phi_{\alpha}\right),\left[H_{\Lambda}\left(\phi_{\alpha}\right)-H_{\Lambda}(\phi),\left[H_{\Lambda}(\phi), A\right]^{(r)}\right]\right]^{(n-r-1)} .
\end{aligned}
$$

Application of Lemma 3.2 with $\Lambda_{i}=\Lambda, i=1, \ldots, n, \phi_{1}=\cdots=\phi_{r}=\phi, \phi_{r+1}=\phi_{\alpha}-\phi$, $\phi_{r+2}=\cdots=\phi_{n}=\phi_{\alpha}$ yields an estimate for the norm of (14), which, together with (12) and $\left\|\phi_{\alpha}\right\| \|_{f} \leqq M$ for some $M$, gives the desired result.

\section{Existence of the Pressure: Definitions and Preparatory Lemmas}

In proving the existence of the limit $\Lambda \rightarrow \infty$ of $P_{\Lambda}$ we shall proceed partly along the same lines as Ruelle [1]. We start considering potentials of finite range.

Definition 4.1. (i) The local range $\Delta(x ; \phi)$ of $\phi$ is given by

$$
\Delta(x ; \phi)=\bigcup_{X \in \mathscr{D}(x ; \phi)} X, \quad \mathscr{D}(x ; \phi)=\left\{X \subset \mathbb{Z}^{v} ; X \ni x, \phi(X) \neq 0\right\} .
$$

(ii) $\phi$ is of locally finite range if

$$
\phi \in \mathscr{B}_{0} \equiv\left\{\psi ; \Delta(x ; \psi) \text { finite for all } x \in \mathbb{Z}^{v}\right\} .
$$

Lemma 4.2. The following conditions are equivalent:

(i) $\bigcup_{x}(\Delta(x ; \psi)-x)$ is finite,

(ii) There exists a finite set $\Delta(\psi)$ and a function $x \mapsto a(x) \in \mathbb{Z}^{v}$ such that $\Delta(x ; \psi) \subset \Delta(\psi)+a(x)$;

(iii) There exists a finite set $\Delta^{\prime}(\psi)$ such that $\psi(X)=0$ unless $X \subset \Delta^{\prime}(\psi)+a(X)$ for a suitable $a(X) \in \mathbb{Z}^{v}$.

Proof. Clearly, (i) implies (ii), (ii) implies (iii). Let us assume (iii). If $X \subset \Delta(x ; \psi)$, then $x \in X, \psi(X) \neq 0$, hence $X \subset \Delta^{\prime}(\psi)+a(X), 0 \in X-x \subset \Delta^{\prime}(\psi)+a(X)-x$, and thus $X-x \subset \Delta^{\prime \prime}(\psi)=\bigcup_{y \in \Delta^{\prime}(\psi)}\left(\Delta^{\prime}(\psi)-y\right), \Delta(x ; \psi)-x \subset \Delta^{\prime \prime}(\psi)$, and $\Delta^{\prime \prime}(\psi)$ is finite because $\Delta^{\prime}(\psi)$ is finite; therefore, (i) holds.

Definition 4.3. $\phi$ is of globally finite range if the conditions of Lemma 4.2 hold. The set of these $\phi$ 's is denoted by $\mathscr{B}_{00}$. 
We are now concerned with potentials $\in \overline{\mathscr{B}}_{f}$ with $f(\xi) \geqq 1 / \xi$. Note that $\overline{\mathscr{B}}_{f}$ is a very large space; it is not at all obvious wether $\mathscr{B}_{0} \cap \overline{\mathscr{B}}_{f}$ is dense in $\overline{\mathscr{B}}_{f}$, certainly, $\mathscr{B}_{00}$ is not

Lemma 4.4. The closure $\overline{\mathscr{B}}_{00}^{f}$ of $\mathscr{B}_{00}$ in $\|\ldots\|_{f}$-norm is the set of all $\phi \in \overline{\mathscr{B}}_{f}$ such that $\sum_{X \ni x}\|\phi(X)\| f(N(X))$ converges uniformly in $x$, i.e. for $\varepsilon>0$, there is a finite $\Lambda_{0}(\varepsilon)$ such that

$$
\sum_{X: x \in X \nsubseteq \Lambda_{0}(\varepsilon)+x}\|\phi(X)\| f(N(X))<\varepsilon .
$$

Proof. Assume uniform convergence, and define

$$
\phi_{\varepsilon}(X)=\left\{\begin{array}{cl}
\phi(X) & \text { if there exists } x \in X: X \subset A_{0}(\varepsilon)+x, \\
0 & \text { otherwise } .
\end{array}\right.
$$

Then it follows that $\phi_{\varepsilon} \rightarrow \phi$ in $\|\ldots\|_{f}$-norm, and $\phi_{\varepsilon}$ satisfies condition (iii) of Lemma 4.2. The other direction is straightforward.

Proposition 4.5. Suppose $\phi, \psi \in \overline{\mathscr{B}}_{f}$ with $f(\xi) \geqq 1 / \xi$.

(i) $\left|P_{\Lambda}(\phi)-P_{\Lambda}(\psi)\right| \leqq \sup _{x \in \Lambda}\|\phi-\psi\|_{f}^{x} \leqq\|\phi-\psi\|_{f}$.

(ii) The function $P_{\Lambda}(\cdot)$ is convex on $\overline{\mathscr{B}}_{f}$.

(iii) $\left|P_{\Lambda}(\phi)-\log d\right| \leqq\|\phi\|_{f}, d=\operatorname{dim} \mathscr{H}_{x}$.

This is nothing but the content of Propositions 2.2.2 and 2.2.3 of [1] stated for potentials in $\mathscr{\mathscr { B }}_{f}$; the proofs of [1] remain valid because we have

$$
\begin{aligned}
\left\|H_{\Lambda}(\phi)\right\| & =\left\|\sum_{x \in \Lambda} \sum_{X: x \in X \subset A} \phi(X) N(X)^{-1}\right\| \\
& \leqq \sum_{x \in \Lambda}\|\phi\|_{f}^{x} \leqq N(\Lambda)\|\phi\|_{f} \quad \text { if } \quad f(\xi) \geqq 1 / \xi .
\end{aligned}
$$

This is the analog of (2.9) in [1], the other equations and conclusions of the proofs in [1] need not be changed. Now we restrict ourselves to interactions of globally finite range. Proposition 2.3.2 of [1] then reads, with $\Delta(\phi)$ as in Lemma 4.2 (ii),

Lemma 4.6. For $\phi \in \mathscr{B}_{00}, \Lambda_{1} \cap \Lambda_{2}=\emptyset$, let $N\left(\Lambda_{1}, \Lambda_{2}\right)$ be the number of points $a \in \mathbb{Z}^{v}$ such that $\Lambda_{1} \cap(\Delta(\phi)+a) \neq \emptyset$ and $\Lambda_{2} \cap(\Delta(\phi)+a) \neq \emptyset$, then

$$
\begin{aligned}
& \left|N\left(\Lambda_{1} \cup \Lambda_{2}\right) P_{\Lambda_{1} \cup \Lambda_{2}}(\phi)-N\left(\Lambda_{1}\right) P_{\Lambda_{1}}(\phi)-N\left(\Lambda_{2}\right) P_{\Lambda_{2}}(\phi)\right| \\
& \quad \leqq\left\|H_{\Lambda_{1} \cup \Lambda_{2}}(\phi)-H_{\Lambda_{1}}(\phi)-H_{\Lambda_{2}}(\phi)\right\| \leqq N\left(\Lambda_{1}, \Lambda_{2}\right)\|\phi\|_{1 / \xi} .
\end{aligned}
$$

The proof is the same as in [1] since it is based on (15).

We consider the box

$$
\Lambda(a)=\left\{x \in \mathbb{Z}^{v} ;-a^{i} \leqq x^{i}<a^{i}, \quad i=1, \ldots, v,\left(a^{1}, \ldots, a^{v}\right) \in \mathbb{Z}^{v}\right\},
$$

and its mutually disjoint translates $\Lambda_{j}, j=1,2, \ldots$, which constitute a covering of $\mathbb{Z}^{\nu}$. Our definition of $\Lambda(a)$ is slightly different from the definition in [1] because we want to have $\Lambda(a)_{R} \infty$ as $a \rightarrow \infty$ (i.e. $a^{x} \rightarrow \infty, x=1, \ldots, v$ ). Let $N_{a}^{-}(\Lambda)$ (resp. $N_{a}^{+}(\Lambda)$ ) denote the number of translates contained in (resp. with non-empty intersection with) $\Lambda$. If $\Lambda \rightarrow \vec{H} \infty$, then, by definition,

$$
N_{a}^{-}(\Lambda) \rightarrow \infty \quad \text { and } \quad N_{a}^{-}(\Lambda) / N_{a}^{+}(\Lambda) \rightarrow 1
$$


If we choose $a$ and $\Lambda$ large enough, we may conclude from (16) as in the proof of Proposition 2.3.2 of [1] that

$$
\left|P_{\Lambda}(\phi)-N_{a}^{-}(\Lambda)^{-1} \sum_{j=1}^{N_{a}^{-}(\Lambda)} P_{\Lambda_{j}}(\phi)\right|<\varepsilon, \quad \phi \in \mathscr{B}_{00} .
$$

For translation covariant potentials, we would have $P_{\Lambda_{\mathrm{j}}}(\psi)=P_{\Lambda(a)}(\psi)$, and (18) would read $\left|P_{\Lambda}(\psi)-P_{\Lambda(a)}(\psi)\right|<\varepsilon$ demonstrating the existence of $\lim _{\Lambda \rightarrow \infty} P_{\Lambda}(\psi)$. This is the point where we need a condition ensuring that the following holds:

(M) $\lim _{\substack{N \rightarrow \infty \\ a \rightarrow \infty}} N^{-1} \sum_{j=1}^{N} P_{\Lambda_{j}}(\phi)$ exists for suitable sequences $\left\{\Lambda_{j}\right\}$ of disjoint translates of $\Lambda(a)$ and for $a \rightarrow \infty$.

In the following section, we shall demonstrate it to hold for RSI-potentials, and in Section VI for ATC-potentials. (M) in turn ensures the existence of $\lim _{\Lambda \rightarrow \infty} P_{\Lambda}(\phi)$. This holds for finite range potentials $\phi \in \mathscr{B}_{00}$. Due to Proposition 4.5, the functions $P_{\Lambda}(\phi)$ are convex and equicontinuous on $\overline{\mathscr{B}}_{1 / \xi}$. Hence we have

Lemma 4.7. Let $\lim _{\Lambda \vec{H} \infty} P_{\Lambda}(\phi)$ exist for $\phi \in \mathscr{D} \subset \mathscr{B}_{00}$. Then $P_{\Lambda}(\phi)$ converge for all $\phi \in \overline{\mathscr{D}}^{f}, f(\xi) \geqq 1 / \xi$, to a convex $\|\ldots\|_{f}$-norm continuous function $P(\phi)$ on $\overline{\mathscr{D}}^{f}$.

Here, $\overline{\mathscr{D}}^{f}$ denotes the $\|\ldots\|_{f}$-norm closure of $\mathscr{D}$.

\section{The Case of the RSI-Potentials}

Let $\phi=\phi_{T}+\phi_{R}$ be a potential consisting of a translation covariant part $\phi_{T}$ and a RSI-potential $\phi_{R}$ as given by Definition 2.2. We have to make precise what is meant by "random distribution" of a subset $I$ in an infinite lattice $\mathbb{Z}^{\nu}$. Let $\Lambda$ be any finite set, and let us consider the distributions $d(\Lambda+a)$ of impurities in $\Lambda+a, a \in \mathbb{Z}^{v}$

$$
d(\Lambda+a)=(\Lambda+a) \cap I .
$$

Definition 5.1. Two distributions $d=d(\Lambda+a), d^{\prime}=d\left(\Lambda+a^{\prime}\right)$ are called of the same type, $d \sim d^{\prime}$, if $d(\Lambda+a)-a=d\left(\Lambda+a^{\prime}\right)-a^{\prime}$.

Given $\Lambda$, there are $2^{N(\Lambda)}$ possible different types $d_{\Lambda}^{(r)}$ of distributions, $r=1,2, \ldots$ $\ldots, 2^{N(\Lambda)}$. If $I$ is distributed at random, we expect every type $d_{\Lambda}^{(r)}$ to occur with a well-defined probability if we look at an arbitrary translate $\Lambda+a$ of $\Lambda$. Probability is used here in the sense of relative frequency of occurrence as it is common in physics. To be precise we shall assume the following:

(R) Let $\left\{\Lambda_{j}\right\}_{j=0,1,2, \ldots}$ be a covering of $\mathbb{Z}^{v}$ by mutually disjoint translates $\Lambda_{j}=\Lambda(a)+a_{j}$ of the box $\Lambda(a)$ defined in (17). Let $N(r ; a ; k)$ be the number of $a_{j}$ 's, $j \leqq k$, with $d\left(\Lambda(a)+a_{j}\right)-a_{j}=d_{\Lambda(a)}^{(r)}$. Then the following limit exists:

$$
\lim _{k \rightarrow \infty}(1 / k) N(r ; a ; k)=v(r ; a), \quad r=1,2, \ldots, 2^{N(\Lambda(a))} .
$$


This completes the definition of RSI-potentials. It is sufficient to require (19) for those cases where $\Lambda^{k}=\bigcup_{j=1}^{k} \Lambda_{j}$ form a $H-R$-sequence. We shall always assume that without further mentioning. For the sake of simplicity, we suppose (R) to be fulfilled for all $a \in \mathbb{Z}^{v}$ although we could do with an infinite sequence $a_{n} \rightarrow \infty$ in most cases.

The essential consequence of $(\mathrm{R})$ is

Lemma 5.2. Let the function $f(\Lambda)$ depend only on the shape of $\Lambda$ and on the type of the impurity distribution $d(\Lambda)$, i.e.

then

$$
f(\Lambda+a)=\tilde{f}(d(\Lambda+a)-a)
$$

$$
\lim _{k \rightarrow \infty} 1 / k \sum_{j=1}^{k} f\left(\Lambda(a)+a_{j}\right)=\sum_{r=1}^{2 N(\Lambda)} v(r ; a) \tilde{f}\left(d_{\Lambda(a)}^{(r)}\right) .
$$

The proof is trivial.

Notice that the sum of two RSI-potentials $\phi_{R}^{1}$ and $\phi_{R}^{2}$, with respective impurity sets $I_{i}$ and respective probabilities $v_{i}(r ; a), i=1,2$, is not a RSI-potential in the sense of Definition 2.2. Nevertheless, we can add them without loosing the important Lemma 5.2. (20) has to be replaced by

$$
f(\Lambda+a)=\tilde{f}\left(d_{1}(\Lambda+a)-a, d_{2}(\Lambda+a)-a\right), d_{i}(\Lambda)=\Lambda \cap I_{i}, \quad i=1,2 ;
$$

and in $(21), v(r ; a)$ has to be replaced by $v(r ; s ; a)=v(r ; a) \cdot v(s ; a)$, with the summation running over $1 \leqq r, s \leqq 2^{N(A)}$. The generalization to more than two sets $I_{i}$ is obvious; we have

Lemma 5.3. Let the function $f(\Lambda)$ depend only on the shape of $\Lambda$ and on the types of the impurity distributions $d_{i}(\Lambda), i=1, \ldots, n$. Here, $d_{i}(\Lambda)=\Lambda \cap I_{i}$; and $I_{i}, i=1, \ldots, n$, are supposed to fulfill (R). Then the limit

exists.

$$
\lim _{k \rightarrow \infty} 1 / k \sum_{j=1}^{k} f\left(\Lambda(a)+a_{j}\right)
$$

If we want to consider more than one kind of impurity, we not only have to add two or more RSI-potentials but have also to introduce an interaction between different impurities. This leads to a more general definition of RSI-potentials.

Definition 5.4. Let $I_{i} \subset \mathbb{Z}^{v}, i=1, \ldots, k$, be randomly distributed sets, i.e. fulfilling (R). Let $d_{i}(\Lambda)=\Lambda \cap I_{i}$ denote the distribution of the $i$-th impurity. $\phi$ is called a generalized RSI-potential if

$$
d_{i}(X)+a=d_{i}(X+a), \quad i=1, \ldots, k, \quad \text { implies } \phi(X+a)=\tau_{a} \phi(X) .
$$

This is a fairly comprehensive class of potentials:

(i) Any translation covariant potential is in that class: take $k=1$ and $I_{1}=\mathbb{Z}^{v}$.

(ii) The sum of two generalized RSI-potentials is again a generalized RSIpotential (with a possibly larger set of impurity sets $I_{j}$ ).

(iii) Trivially, a RSI-potential is a generalized RSI-potential. 
(iv) A potential of the following kind is a generalized RSI-potential: $\phi=\phi_{T}+\phi_{R}^{1}+\phi_{R}^{2}+\phi_{12}$, with a translation covariant part $\phi_{T}$, two RSI-potentials $\phi_{R}^{i}$ and a potential $\phi_{12}$ describing the interaction of the two impurities, where $\phi_{12}(X)=0$ unless $X=X_{1} \cup X_{2}$ and $X_{1} \subset I_{1}, X_{2} \subset I_{2}$, provided $\phi_{12}$ fulfils (23) for $i=1,2$, [i.e. a relation similar to (9)].

Now we have to make sure that our potentials have finite norm and, moreover, can be approximated by potentials of globally finite range. For RSI-potentials, we have the following results:

Lemma 5.5. Let $\phi_{R}=\sum_{x \in I} \phi^{x}$ be a RSI-potential. If $\phi^{x_{0}} \in \overline{\mathscr{B}}_{\xi f(\xi)}$ for any $x_{0} \in I$, then $\phi_{R} \in \overline{\mathscr{B}}_{f(\xi)}$.

Lemma 5.6. Let $0 \in I$ and assume that $\phi^{0}$ is centered around 0 .

(i) Let $\phi^{0}$ be of locally finite range, then $\phi_{R}, \phi_{R}(X)=\sum_{x \in I} \tau_{x} \phi^{0}(X-x)$, is of globally finite range.

(ii) Any $\phi^{0} \in \overline{\mathscr{B}}_{f_{1}}$ can be approximated in $\|\ldots\|_{f_{1}}$-norm by finite range potentials $\phi_{\alpha}^{0}$ centered around 0 .

(iii) If $\left\|\phi_{\alpha}^{0}-\phi^{0}\right\|_{\xi f(\xi)} \rightarrow 0$ then $\left\|\phi_{R_{\alpha}}-\phi_{R}\right\|_{f} \rightarrow 0, \phi_{R_{\alpha}}(X)=\sum_{x \in I} \tau_{x} \phi_{\alpha}^{0}(X-x)$.

The proofs of these Lemmas will be left to the reader. As a consequence of Lemma 5.6, a potential $\phi_{R}$ as defined in Lemma 5.5 is $\in \overline{\mathscr{B}}_{00}^{f}$, where $\overline{\mathscr{B}}_{00}^{f}$ denotes the $\|\ldots\|_{f}$-norm closure of $\mathscr{B}_{00}$. If $\phi_{R}^{W}$, defined in (8) and (9), is - in the sense of (10) - derived from a potential $\phi^{W} \in \mathscr{B}_{f}^{T}$, it follows trivially that also $\phi_{R}^{W} \in \overline{\mathscr{B}}_{00}^{f}$; furthermore, the approximating potentials $\in \mathscr{B}_{00}$ are also RSI-potentials. As regards arbitrary generalized RSI-potentials, we take this property as an additional assumption; henceforth we consider potentials given by the following definition:

\section{Definition 5.7. $\overline{\mathscr{B}}_{R}^{f}=\overline{\left\{\phi \in \mathscr{B}_{00} ; \phi \text { generalized RSI-potential }\right\}}$.}

$\{\ldots\}^{f}$ denotes the $\|\ldots\|_{f}$-norm closure of $\{\ldots\} . \overline{\mathscr{B}}_{R}^{f}$ is a closed linear subspace of $\overline{\mathscr{B}}_{00}^{f}$. Collecting our previous results we are now able to prove

Theorem 5.8. Assume $\phi \in \overline{\mathscr{B}}_{R}^{f}, f(\xi) \geqq 1 / \xi$. Then $\lim _{\Lambda \rightarrow \infty} P_{\Lambda}(\phi)=P(\phi)$ exists if $\Lambda \rightarrow \infty$ in the sense of van Hove and Robinson, and it is independent of the chosen sequence $\Lambda . P(\phi)$ is a convex continuous function on $\overline{\mathscr{B}}_{R}^{f}$.

Proof. Suppose $\phi \in \overline{\mathscr{B}}_{R}^{f} \cap \mathscr{B}_{00}$. Because of (23),

$$
P_{A}(\phi)=N(\Lambda)^{-1} \log \operatorname{Tr}_{\Lambda} \exp \left(-H_{\Lambda}(\phi)\right)
$$

does not depend on where $\Lambda$ is situated but only on the shape of $\Lambda$ and on the types of $d_{i}(\Lambda)$. Application of Lemma 5.3 then ensures the existence of

$$
\lim _{k \rightarrow \infty} 1 / k \sum_{j=1}^{k} P_{\Lambda},(\phi)=P(a ; \phi),
$$

where $\Lambda_{j}$ are disjoint translates of $\Lambda(a)$. Let $\Lambda_{H, R} \infty$, then the sequence of $\Lambda_{j}$ 's contained in $\Lambda$ eventually yields a covering of $\mathbb{Z}^{v}$; and (18) and (24) give, for sufficiently large $a_{0}(\varepsilon)$ and sufficiently large $\Lambda_{0}(\varepsilon ; a)$,

$$
\left|P_{\Lambda}(\phi)-P(a ; \phi)\right|<2 \varepsilon \quad \text { if } \quad a>a_{0}(\varepsilon), \quad \Lambda \supset \Lambda_{0}(\varepsilon ; a) .
$$


Let $a_{1}, a_{2}$ be larger than $a_{0}(\varepsilon / 4)$, and choose $\Lambda \supset \Lambda_{0}\left(\varepsilon / 4, a_{1}\right) \cup \Lambda_{0}\left(\varepsilon / 4, a_{2}\right)$, then $\left|P\left(a_{1} ; \phi\right)-P\left(a_{2} ; \phi\right)\right|<\varepsilon$, hence $\lim _{\Lambda \rightarrow \vec{R} \infty} P_{\Lambda}(\phi)$ exists.

The rest follows from Lemma 4.7 .

Remark 5.9. Notice that in $(\mathrm{R})$ we did not make any assumption about the magnitude of the probability of a given type of impurity distribution for a fixed $\Lambda(a)$. (In speaking of a "random" distribution, one could try to justfy the requirement that $d_{\Lambda(a)}^{(r)}$ and $d_{\Lambda(a)}^{\left(r^{\prime}\right)}$ occur with the same probability if they contain the same number of impurity sites.) This leaves open the possibility of a superimposed partial regularity of the impurities which could - in a real crystal - be caused by an interaction between them. Actually, even a completely regular distribution of impurities, i.e. a $\phi_{R}$ exhibiting covariance under a subgroup of lattice translations, fulfils (R). This case can already be treated in the usual framework of covariant potentials if one considers the periodicity cells as elements of a new lattice $\tilde{\mathbb{Z}}^{v}$. Clearly, the outcome has be the same as above (see Appendix).

\section{Locally Disturbed Potentials}

If there is only a local concentration of impurities, in other words, if the potential is disturbed locally by an operator, say, $\in \mathscr{A}_{\Lambda_{0}}$, one does not expect a change in the pressure as $\Lambda$ goes to infinity. Indeed, if we consider asymptotically translation covariant potentials $\phi \in \mathscr{B}_{f}^{a}$ (see Definition 2.1), then the following results hold:

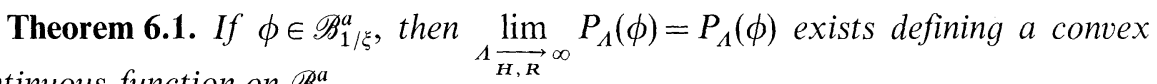
continuous function on $\mathscr{B}_{1 / \xi}^{a}$.

Definition 6.2. $\phi_{1}$ and $\phi_{2}$ are said to be $f$-asymptotically equal, $\phi_{1} \tilde{f}_{2} \phi_{2}$, if, for $\varepsilon>0$, there is a $\tilde{\Delta}_{\varepsilon}$ such that $\left\|\phi_{1}-\phi_{2}\right\|_{f}^{x}<\varepsilon$ for $x \notin \tilde{\Delta}_{\varepsilon}$.

Theorem 6.3. Suppose $\phi_{1} \underset{f}{\phi_{2}}, \phi_{1} \in \overline{\mathscr{B}}_{f}$ and $\phi_{2} \in \mathscr{B}_{f}^{a}, f(\xi) \geqq 1 / \xi$. Then

(i) $\phi_{1} \in \mathscr{B}_{f}^{a}$,

(ii) $P\left(\phi_{1}\right)=P\left(\phi_{2}\right)$.

Corollary 6.4. If the translation covariant potential $\phi$ is "locally disturbed" by $\phi^{\prime}$, i.e. $\phi^{\prime} \in \mathscr{B}_{f}^{a}, \phi \widetilde{f} \phi+\phi^{\prime}$, then $P\left(\phi+\phi^{\prime}\right)=P(\phi)$.

Proof of Theorem 6.1. We first take $\phi \in \mathscr{B}_{00} \cap \mathscr{B}_{f}^{a}$. Combination of Definition 2.1 and Proposition 4.5 gives

$$
\left|P_{\Lambda+c}(\phi)-P_{\Lambda}(\phi)\right|<\varepsilon
$$

provided $\Lambda$ and $\Lambda+c$ lie outside $\Delta_{\varepsilon}(\phi)$. Now let us choose a large $a$ and a sufficiently large $\Lambda$ such that $\Delta_{\varepsilon}(\phi) \subset \Lambda(a)$ and $\Lambda \supset \Lambda_{1} \cup \Lambda_{2}$, where $\Lambda_{1}=\Lambda(a)$, $\Lambda_{2}=\Lambda(a)+2 a$. Clearly, all translates $\Lambda_{j}$ of $\Lambda(a), \Lambda_{j} \subset \Lambda, j=2, \ldots, N_{a}^{-}(\Lambda)$, lie outside $\Delta_{\varepsilon}(\phi)$, hence we can apply (25) to $\Lambda_{2}$ and $\Lambda_{j}, j>2$. The role of $N_{a}^{-}(\Lambda)^{-1} \Sigma P_{\Lambda_{j}}(\phi)$ in (18) can now be taken over by $P_{\Lambda_{2}}(\phi)$; consequently, as is easily seen, $\lim _{\Lambda \rightarrow \boldsymbol{H}_{\boldsymbol{R}}} P_{\Lambda}(\phi)$ exists. The rest follows from Lemma 4.7 and

Proposition 6.5. $\mathscr{B}_{00} \cap \mathscr{B}_{f}^{a}$ is $\|\ldots\|_{f}$-norm dense in $\mathscr{B}_{f}^{a}$.

The proof is a bit clumsy but straightforward, and is left to the reader. 
Remark 6.6. For the proof of the existence of $P(\phi), \phi \in \mathscr{B}_{00}$, we could have done with a weaker definition of asymptotical translation covariance, for instance, by assuming $\left\|\sum_{X \subset A}\left(\phi(X+a)-\tau_{a} \phi(X)\right)\right\| \leqq N(\Lambda) \varepsilon$ if $\Lambda$ and $\Lambda+a$ are outside some $\Delta_{\varepsilon}^{\prime}(\phi)$. But this is not sufficient to prove Proposition 6.5.

Proof of Theorem 6.3. (i) $\phi_{2}-\phi_{1}$ is readily seen to fulfil Eq. (5) of Definition 2.1, hence $\phi_{1}=\phi_{2}-\left(\phi_{2}-\phi_{1}\right) \in \mathscr{B}_{f}^{a}$ because $\mathscr{B}_{f}^{a}$ is a linear subset of $\overline{\mathscr{B}}_{f}$.

(ii) According to Proposition 6.5, $\phi_{1}$ and $\phi_{2}$ can be approximated by $\phi_{1 \alpha}$ and $\phi_{2 \alpha}$, respectively, $\phi_{1 \alpha}, \phi_{2 \alpha} \in \mathscr{B}_{00} \cap \mathscr{B}_{f}^{a}$. If $a$ is large enough, we have $\Lambda_{2} \cap \tilde{\Delta}_{\varepsilon}$ $=(\Lambda(a)+2 a) \cap \tilde{\Delta}_{\varepsilon}=\emptyset$ and therefore

$$
\left|P_{\Lambda_{2}}\left(\phi_{1 \alpha}\right)-P_{\Lambda_{2}}\left(\phi_{2 \alpha}\right)\right| \leqq \sup _{x \in A_{2}}\left\|\phi_{1 \alpha}-\phi_{2 \alpha}\right\|_{f}^{x}<3 \varepsilon .
$$

Reasoning as in the proof of Theorem 6.1 we get the desired result.

Remark 6.7. It is to be expected that a theorem similar to Corollary 6.4 also holds for a generalized RSI-potential disturbed by $\phi^{\prime} \in \mathscr{B}_{f}^{a}, \phi^{\prime} \widetilde{f} 0$. In this case, the role of $P_{\Lambda_{2}}(\phi)$ is taken over by $\lim _{k \rightarrow \infty} 1 / k \sum_{j=1}^{k} P_{\Lambda_{j}}(\phi)$, where $\left\{\Lambda_{j}\right\}_{j=0,1,2, \ldots}$ is a covering of $\mathbb{Z}^{v}$. But we can do with a covering of $\mathbb{Z}^{v}-Y$, where $Y$ is a suitable finite set containing $\tilde{\Delta}_{\varepsilon}$, and argue along the same lines as in the proof of Theorem 6.3 .

\section{Extremality Property of $\boldsymbol{P ( \phi )}$}

It is well known [3, see also 4] that $P(\phi), \phi \in \mathscr{B}_{f}^{T}$, can be obtained by a variational principle:

$$
P(\phi)=\sup _{\varrho \in \mathscr{S}_{0}}\left(s(\varrho)-\varrho\left(A_{\phi}\right)\right) .
$$

Here, $A_{\phi}=\sum_{X \ni 0} \phi(X) N(X)^{-1}$ is the observable of the mean energy per site and $s(\varrho)$ is the entropy density,

$$
s(\varrho)=\lim _{\substack{\Lambda \rightarrow \infty \\ \mathrm{H}^{\infty}}} S_{\varrho}(\Lambda) / N(\Lambda), \quad S_{\varrho}(\Lambda)=-\operatorname{Tr}_{\Lambda} \varrho_{\Lambda} \log \varrho_{\Lambda} .
$$

$S_{\varrho}(\Lambda)$ is well defined for arbitrary $\varrho \in \mathscr{S}$, but the limit in (27) is only known to exist for $\varrho \in \mathscr{S}_{0}$ (see, for instance, [1], where (27) is shown with $\Lambda=\Lambda(a) \rightarrow \infty$, the generalization is due to $[5,6])$. Since we want to consider non-covariant potentials, we cannot expect from the very beginning that an invariant equilibrium state can be found. There is a second difficulty in proving a variational principle: $A_{\phi}$, as defined above, is the observable of the energy density only for translation covariant potentials. In general, it has to be replaced by $H_{\Lambda}(\phi) / N(\Lambda)$ or by $A_{\phi}(\Lambda)=N(\Lambda)^{-1} \sum_{x \in \Lambda} A_{\phi}^{x}, A_{\phi}^{x}=\sum_{X \ni x} \phi(X) / N(X)$, and, of course, the limit $\Lambda \rightarrow \infty$ has to be taken at a suitable moment in the computation. One readily checks - for instance, with the help of a modification of Lemma 3.8 in [7] - that $\| A_{\phi}(\Lambda)$ $-H_{\Lambda}(\phi) / N(\Lambda) \|$ tends to zero as $\Lambda \underset{H, R}{\longrightarrow} \infty$, provided $\phi \in \overline{\mathscr{B}}_{1 / \xi}$; therefore, it does not matter which expression is chosen. Unfortunately, $\lim _{\Lambda \rightarrow \infty} \varrho\left(H_{\Lambda}(\phi) / N(\Lambda)\right)$ need 
not exist. We define the entropy density and the energy density for arbitrary states and arbitrary potentials by

$$
\begin{array}{r}
\text { Definition 7.1. } \bar{s}(\varrho)=\sup _{\{\Lambda\}_{H . R}} \limsup _{\Lambda \rightarrow \infty} S_{\varrho}(\Lambda) / N(\Lambda), \\
E(\varrho ; \phi)=\sup _{\{\Lambda\}_{H, R}} \lim \sup _{\Lambda \rightarrow \infty} \varrho\left(H_{\Lambda}(\phi) / N(\Lambda)\right) .
\end{array}
$$

sup denotes the supremum over all $H-R$-sequences. $\{\Lambda\}_{H, R}$

If $\varrho \in \mathscr{S}_{0}$, then (i) $\bar{S}(\varrho)=s(\varrho)$; (ii) $\lim _{\Lambda, \bar{H}_{R}} \varrho\left(H_{\Lambda}(\phi) / N(\Lambda)\right)$ exists if $\phi \in \overline{\mathscr{B}}_{R}^{f}$ and equals $E(\varrho ; \phi)$; (iii) $E(\varrho ; \phi)=\varrho\left(A_{\phi}\right)$, provided $\phi$ is translation covariant. In a recent paper [8], Lima and Naudts have introduced non-covariant potentials which are "uniformly averageable". For these potentials, they define the mean energy in a state $\varrho \in \mathscr{P}_{0}$ by $\lim _{\Lambda \rightarrow \infty} \varrho\left(A_{\phi}(\Lambda)\right)$. This clearly coincides with our definition. The condition of being uniformly averageable seems to be more restrictive than our conditions on $\phi$, nevertheless, their class of potentials does not have an obvious connection with our set $\overline{\mathscr{B}}_{R}^{f}$.

The quantities $E$ and $\bar{s}$ can be used to state a generalized variational principle:

Theorem 7.2. Assume $\phi \in \overline{\mathscr{B}}_{R}^{f}, f(\xi) \geqq 1 / \xi$. By Theorem 5.8, $\underset{\Lambda}{\underset{H, R}{\longrightarrow} \infty} P_{\Lambda}(\phi)=P(\phi)$ exists, $P(\phi)$ being independent of the chosen sequence. $P(\phi)$ is given by

$$
P(\phi)=\sup _{\varrho \in \mathscr{S}}(\bar{S}(\varrho)-E(\varrho ; \phi)) \text {. }
$$

Proof. We proceed in three steps: first, we show

$$
P(\phi) \geqq \sup _{\varrho \in \mathscr{S}}(\bar{S}(\varrho)-E(\varrho ; \phi)) ;
$$

second, we exhibit a set of states $\varrho^{a}, a \in \mathbb{Z}^{v}$, such that

$$
N(\Lambda)^{-1}\left(S_{\varrho^{a}}(\Lambda)\right)-\varrho^{a}\left(H_{\Lambda}(\phi)\right) \geqq P(\phi)-\varepsilon_{a}
$$

for all sufficiently large members $\Lambda$ of a suitable $H-R$-sequence $\{\Lambda\}$, where $\varepsilon_{a}$ can be made arbitrarily small for large $a$; and finally, we demonstrate that, for these states, the following holds:

$$
\bar{S}\left(\varrho^{a}\right)=\lim _{\Lambda, R} N(\Lambda)^{-1} S_{\varrho^{a}}(\Lambda), \quad E\left(\varrho^{a} ; \phi\right)=\lim _{H, \boldsymbol{R}} \varrho^{a}\left(H_{\Lambda}(\phi) / N(\Lambda)\right),
$$

independent of the chosen $H-R$-sequence. From this last statement, together with (30), we get

$$
\bar{S}\left(\varrho^{a}\right)-E\left(\varrho^{a} ; \phi\right) \geqq P(\phi)-\varepsilon_{a},
$$

and hence the converse of (29), which then completes the proof of Theorem 7.2.

Proof of (29). With the help of the concavity of the logarithm and Peierls' inequality, one easily checks $S_{\varrho}(\Lambda)-\varrho\left(H_{\Lambda}(\phi)\right) \leqq \log \operatorname{Tr}_{\Lambda} e^{-H_{\Lambda}(\phi)}$. Therefore

$$
\begin{aligned}
\bar{s}(\varrho)-E(\varrho ; \phi) & \leqq \sup _{\{\Lambda\}_{H, R}} \limsup _{\Lambda \rightarrow \infty} N(\Lambda)^{-1}\left[S_{\varrho}(\Lambda)-\varrho\left(H_{\Lambda}(\phi)\right)\right] \\
& \leqq \sup _{\{\Lambda\}_{H, R}} \limsup _{\Lambda \rightarrow \infty} N(\Lambda)^{-1} \log \operatorname{Tr}_{\Lambda} e^{-H_{\Lambda}(\phi)}=\sup _{\{\Lambda\}_{H, R}} \limsup _{\Lambda \rightarrow \infty} P_{\Lambda}(\phi) .
\end{aligned}
$$


Because $\lim _{\Lambda, R} P_{\Lambda}(\phi)=P(\phi)$ exists independent of the chosen sequence, $\sup _{\{\Lambda\}_{H, R}} \limsup _{\Lambda \rightarrow \infty} P_{\Lambda}(\phi)$ and $\lim _{\Lambda, \underset{H, R}{\infty}} P_{\Lambda}(\phi)$ coincide yielding (29), since (32) holds for arbitrary $\varrho \in \mathscr{S}$.

Proof of (30). Let again $\left\{\Lambda_{j}\right\}_{j=1,2, \ldots}$ be a covering of $\mathbb{Z}^{v}$, where $\Lambda_{j}=\Lambda(a)+a_{j}$ are mutually disjoint translates of the box $\Lambda(a) ; a_{1}=0$. We define a state $\varrho^{a}$ by prescribing its density matrices $\varrho_{\Lambda}^{a}$ for $\Lambda=\Lambda_{j}$ and for $\Lambda=$ finite union of some $\Lambda_{j}$ :

$\varrho_{\Lambda_{j}}^{a}=\frac{\exp \left(-H_{\Lambda_{j}}(\phi)\right)}{\operatorname{Tr}_{\Lambda_{j}} \exp \left(-H_{\Lambda_{j}}(\phi)\right)}, \quad \varrho_{\Lambda}^{a}=\prod_{j \in J}^{\otimes} \varrho_{\Lambda_{j}} \quad$ if $\quad \Lambda=\bigcup_{j \in J} \Lambda_{j}, \quad J$ finite .

We now consider a $H-R$-sequence $\{\Lambda\}$ where the $\Lambda$ 's are composed of translates $\Lambda_{j}$. [It is sufficient to show (30) for a suitable sequence.] We then find

and, consequently,

$$
S_{\varrho^{a}}\left(\bigcup_{j \in J} \Lambda_{j}\right)=\sum_{j \in J} S_{\varrho^{a}}\left(\Lambda_{j}\right)
$$

$$
S_{\varrho^{a}}\left(\bigcup_{j \in J} \Lambda_{j}\right) / N\left(\bigcup_{j \in J} \Lambda_{j}\right)=k^{-1} \sum_{j=1}^{k} S_{\varrho^{a}}\left(\Lambda_{j}\right) / N\left(\Lambda_{j}\right),
$$

where $k$ is the cardinality of $J$. Let us write $S_{\varrho^{a}}\left(\Lambda_{j}\right)=-\operatorname{Tr}_{\Lambda_{j}} \varrho_{\Lambda_{j}}^{a} \log \varrho_{\Lambda_{j}}^{a}=\operatorname{Tr}_{\Lambda_{j}} \varrho_{\Lambda_{j}}^{a} H_{\Lambda_{j}}(\phi)$ $+N\left(\Lambda_{j}\right) P_{\Lambda_{j}}(\phi)$ and insert it into (34):

$$
S_{\varrho^{a}}\left(\cup \Lambda_{j}\right) / N\left(\cup \Lambda_{j}\right)=\varrho^{a}\left(k^{-1} \sum_{j=1}^{k} H_{\Lambda_{j}}(\phi) / N\left(\Lambda_{j}\right)\right)+k^{-1} \sum_{j=1}^{k} P_{\Lambda_{j}}(\phi) .
$$

According to (24) and the reasoning thereafter, the second term on the r.h.s. is arbitraily close to $P(\phi)$ if $\phi \in \mathscr{B}_{00}$ and if $a$ and $\Lambda=\cup \Lambda_{j}$ are chosen large enough. Since $P_{\Lambda}(\phi)$ is continuous in $\phi \in \overline{\mathscr{B}}_{1 / \xi}$, uniformly in $\Lambda$, according to Proposition 4.5 , the same is true of $P(\phi)$, and we can replace the condition $\phi \in \mathscr{B}_{00}$ by $\phi \in \overline{\mathscr{B}}_{R}^{f}$. The proof is practically complete if we can show that

$$
\left|\varrho\left(k^{-1} \sum_{j=1}^{k} H_{\Lambda_{j}}(\phi) / N\left(\Lambda_{j}\right)-H_{\Lambda}(\phi) / N(\Lambda)\right)\right|<\varepsilon \quad \text { for large } a \text { and } \Lambda \text {. }
$$

According to the assumptions of Theorem 7.2, $\phi$ can be approximated by some $\phi_{\alpha} \in \mathscr{B}_{00}$. Now remember Eq. (16) and the conclusions leading from (16) to (18). By exactly the same reasoning, we find

$$
N(\Lambda)^{-1}\left\|H_{\Lambda}\left(\phi_{\alpha}\right)-\sum_{j=1}^{k} H_{\Lambda_{j}}\left(\phi_{\alpha}\right)\right\|<\varepsilon
$$

which, together with (15): $\left\|H_{\Lambda}\left(\phi-\phi_{\alpha}\right)\right\| \leqq N(\Lambda)\left\|\phi-\phi_{\alpha}\right\|_{1 / \xi}$ implies (36).

Proof of (31). Instead of (31), we establish a slightly more general proposition.

Proposition 7.3. Assume $\phi, \psi \in \overline{\mathscr{B}}_{R}^{1 / \xi}$ and let $\varrho^{\alpha}$ be given by (33) (i.e. defined with

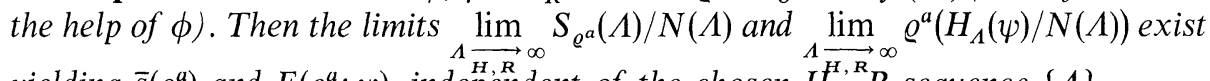
yielding $\bar{S}\left(\varrho^{a}\right)$ and $E\left(Q^{a} ; \psi\right)$, independent of the chosen $H^{H, R}-R$-sequence $\{\Lambda\}$. 
Proof. $a, \phi$, and $\psi$ are fixed throughout the whole proof. Let us first consider $H-R$-sequences $\{\Lambda\}$ where $\Lambda=\cup \Lambda_{j}^{b} . \Lambda_{j}^{b}$ are mutually disjoint translates of $\Lambda(b)$, covering $\mathbb{Z}^{\nu} . b$ is assumed to be an odd multiple of $a, b=(2 m+1) a$, in order that $\Lambda_{j}^{b}$ and $\Lambda$ can also be written as unions of translates $\Lambda_{j}=\Lambda_{j}^{a}$ of $\Lambda(a) ; a$ and $\Lambda_{j}$ are the same as in the definition (33) of $\varrho^{a}$. Notice that $S_{\varrho^{a}}\left(\Lambda_{j}^{b}\right) / N\left(\Lambda_{j}^{b}\right)$ and

$$
\varrho^{a}\left(H_{\Lambda_{j}^{b}}(\psi) / N\left(\Lambda_{j}^{b}\right)\right),
$$

considered as functions of $\Lambda_{j}^{b}$, depend only on the types of the impurity distributions $d_{i}\left(\Lambda_{j}^{b}\right)$ belonging to $\phi$ and $\psi$, i.e. they satisfy the analog of Eq. (22) with $\Lambda$ and $\Lambda+a$ replaced by $\Lambda_{j}^{b}$ and $\Lambda_{j^{\prime}}^{b}$. This suffices to reach the conclusion of Lemma 5.3 with $\Lambda(b)$ in place of $\Lambda(a)$. Therefore, the following limits exist [with $b=a$ and $b=(2 m+1) \cdot a$, respectively $]$ :

and

$$
s^{\prime}\left(\varrho^{a}\right) \equiv \lim _{k \rightarrow \infty} k^{-1} \sum_{j=1}^{k} S_{\varrho^{a}}\left(\Lambda_{j}^{a}\right) / N\left(\Lambda_{j}^{a}\right)
$$

$$
E\left(\varrho^{a} ; \psi ; b\right) \equiv \lim _{k \rightarrow \infty} k^{-1} \sum_{j=1}^{k} \varrho^{a}\left(H_{\Lambda_{j}^{b}}(\psi) / N\left(\Lambda_{j}^{b}\right)\right) \text {. }
$$

Because $\bigcup_{j \in J} \Lambda_{j}^{b}=\bigcup_{j \in J^{\prime}} \Lambda_{j}^{a}$ for some $J^{\prime}$, we conclude from (34) that $\lim _{\Lambda \rightarrow \infty} S_{\varrho^{a}}(\Lambda) / N(\Lambda)$ $=s^{\prime}\left(\varrho^{a}\right)$ for $H-R$-sequences $\left\{\Lambda \in \cup \Lambda_{j}^{b}\right\}, b=(2 m+1) a$.

Invoking the strong subadditivity of $S_{\varrho}(\Lambda)[5,6]$ we get

$$
\lim _{\Lambda \rightarrow \infty} S_{\varrho^{a}}(\Lambda) / N(\Lambda)=s^{\prime}\left(\varrho^{a}\right),
$$

for arbitrary $H-R$-sequences, and due to the independence of the special sequence, $s^{\prime}\left(\varrho^{a}\right)=\bar{s}\left(\varrho^{a}\right)$.

Equation (37) does not only hold for $\Lambda=\cup \Lambda_{j}$, but for an arbitrary sufficiently large $\Lambda$ from a $H-R$-sequence $\{\Lambda\}$ if the summation runs over all translates $\Lambda_{j}$ contained in $\Lambda$; together with (15) (with $\psi-\psi_{\alpha}$ instead of $\phi$ ) we conclude that $E\left(\varrho^{a} ; \psi ; b\right)$ has a limit as $b \rightarrow \infty$, which is independent of the chosen $H-R$-sequence and thus coincides with $E\left(\varrho^{a} ; \psi\right)$.

Theorem 7.2 looks as neat as the corresponding theorem [Eq. (26)] for translation covariant potentials, however, it is not, the reason being that upper semicontinuity in $\varrho$ of $\bar{s}(\varrho)$ cannot readily be shown. Moreover, it is not obvious that $E(\varrho ; \phi)$ is linear in $\phi$ for all $\varrho \in \mathscr{S}$. Let us recall what is known for the translation invariant case $[3,9,10]$.

(a) $s(\varrho)$ is upper semicontinuous on $\mathscr{S}_{0}$, hence the supremum $\sup _{\varrho \in \mathscr{S}_{0}}\left(s(\varrho)-\varrho\left(A_{\phi}\right)\right)$ $=P(\phi), \phi \in \mathscr{B}_{1 / \xi}^{T}$, is attained for some $\varrho^{\phi} \cdot \varrho^{\phi}$ is called an equilibrium state, $s\left(\varrho^{\phi}\right)$ [resp. $\varrho^{\phi}\left(A_{\phi}\right)$ ] is the corresponding equilibrium entropy (resp. energy) density.

(b) A linear functional $\alpha(\psi)$ on $\mathscr{B}_{1 / \xi}^{T}$ is called a tangent functional at $\phi$ to the graph of $P(\cdot)$ if

$$
P(\phi+\psi) \geqq P(\phi)-\alpha(\psi), \quad \phi, \psi \in \mathscr{B}_{1 / \xi}^{T} .
$$

An equilibrium state $\varrho^{\phi} \in \mathscr{S}_{0}$ defines a tangent functional $\alpha^{\phi}$ by $\varrho^{\phi}\left(A_{\psi}\right)=\alpha^{\phi}(\psi)$ and vice versa.

(c) Let $T \subset \mathscr{B}_{1 / \xi}^{T}$ denote the set of potentials such that the tangent functional $\alpha^{\phi}$ at $\phi$ to the graph of $P(\cdot)$ is unique. Then $\alpha^{\phi}=\mathrm{w}_{\Lambda \rightarrow \infty} \lim _{\Lambda} \alpha_{\Lambda}^{\phi}$ where $\alpha_{\Lambda}^{\phi}$ are tangent 
functionals at $\phi$ to the graphs of $P_{\Lambda}(\cdot)$; and

$$
\varrho^{\phi}\left(A_{\psi}\right)=\lim _{\substack{\rightarrow \rightarrow \infty \\ H}} N(\Lambda)^{-1} \operatorname{Tr}_{\Lambda} e^{-H_{\Lambda}(\phi)} H_{\Lambda}(\psi)\left(\operatorname{Tr}_{\Lambda} e^{-H_{\Lambda}(\phi)}\right)^{-1}, \quad \phi, \psi \in \mathscr{B}_{1 / \xi}^{T} .
$$

Now we consider the case of a generalized RSI-potential. Let again $T$ denote the set of those potentials for which the tangent to the graph of $P(\cdot)$ is unique. Since upper semicontinuity of the entropy density is lost, there is no ful counterpart of (a). Nevertheless, we can define the equilibrium entropy (resp. energy) density.

Lemma 7.4. $E(\varrho ; \phi)$ is a continuous function of $\phi$ on $\overline{\mathscr{B}}_{1 / \xi}$.

Proof. From Definition 7.1 we get

and

$$
E\left(\varrho ; \psi_{1}+\psi_{2}\right) \leqq E\left(\varrho ; \psi_{1}\right)+E\left(\varrho ; \psi_{2}\right)
$$

$$
|E(\varrho ; \psi)| \leqq\|\psi\|_{1 / \xi}
$$

because of $\mid \varrho\left(H_{\Lambda}(\psi) / N(\Lambda) \mid \leqq\left\|H_{\Lambda}(\psi) / N(\Lambda)\right\| \leqq\|\psi\|_{1 / \xi}\right.$. Thus it follows that

$$
\begin{aligned}
-\left\|\psi_{2}\right\|_{1 / \xi} & \leqq-E\left(\varrho ;-\psi_{2}\right)=E\left(\varrho ; \psi_{1}+\psi_{2}-\psi_{2}\right)-E\left(\varrho ; \psi_{1}\right)-E\left(\varrho ;-\psi_{2}\right) \\
& \leqq E\left(\varrho ; \psi_{1}+\psi_{2}\right)-E\left(\varrho ; \psi_{1}\right) \\
& \leqq E\left(\varrho ; \psi_{1}\right)+E\left(\varrho ; \psi_{2}\right)-E\left(\varrho ; \psi_{1}\right)=E\left(\varrho ; \psi_{2}\right) \leqq\left\|\psi_{2}\right\|_{1 / \xi},
\end{aligned}
$$

hence $E\left(\varrho ; \psi_{1}+\psi_{2}\right)-E\left(\varrho ; \psi_{1}\right) \rightarrow 0$ as $\psi_{2} \rightarrow 0$ in $\|\ldots\|_{1 / \xi^{-n o r m}}$.

Q.E.D.

For $\varrho=\varrho^{a}$, we have the equality sign in (40) because

$$
E\left(\varrho^{a} ; \psi\right)=\lim _{\Lambda \rightarrow \infty} \varrho^{a}\left(H_{\Lambda}(\psi) / N(\Lambda)\right),
$$

i.e. $E\left(\varrho^{a} ; \psi\right)$ is linear and continuous in $\psi$. Therefore, $\left\{E\left(\varrho^{a} ; \cdot\right) ; a \in \mathbb{Z}^{v}\right\}$ is a relatively compact set in the dual $\left(\overline{\mathscr{B}}_{R}^{f}\right)^{*}$ of $\overline{\mathscr{B}}_{R}^{f} . P(\cdot)$ is considered as a function over $\overline{\mathscr{B}}_{R}^{f}$, and, as usual $f(\xi) \geqq 1 / \xi$. With this in mind, we can formulate what can be proved instead of (a), (b), and (c):

Proposition 7.5. Let $\varrho^{a}$ be defined by (33) with a generalized RSI-potential $\phi$.

(i) Any point of accumulation of $\left\{E\left(\varrho^{a} ; \cdot\right) ; a \in \mathbb{Z}^{\nu}\right\}$ defines a tangent functional $E^{\phi}(\psi)$ at $\phi$ to the graph of $P(\cdot)$.

(ii) To every $E^{\phi}(\psi)$, there corresponds a point of accumulation $s^{\phi}$ of $\left\{\bar{s}\left(\varrho^{a}\right)\right.$; $\left.a \in \mathbb{Z}^{\nu}\right\}$, and

$$
P(\phi)=s^{\phi}-E^{\phi}(\phi)
$$

(iii) If $\phi \in T$, then $E^{\phi}(\cdot)$ and $s^{\phi}$ are unique, and $E^{\phi}(\psi)=\lim _{\Lambda \rightarrow \vec{R}} \alpha_{\Lambda}^{\phi}(\psi)$, where $\alpha_{\Lambda}^{\phi}$ are tangent functionals at $\phi$ to the graphs of $P_{\Lambda}(\cdot)$; furthermore,

$$
\begin{aligned}
E^{\phi}(\psi) & =\lim _{\Lambda \rightarrow,} N(\Lambda)^{-1} \operatorname{Tr}_{\Lambda} e^{-H_{\Lambda}(\phi)} H_{\Lambda}(\psi)\left(\operatorname{Tr}_{\Lambda} e^{-H_{\Lambda}(\phi)}\right)^{-1} \\
& =\lim _{a \rightarrow \infty} \varrho^{a}\left(H_{\Lambda(a)}(\psi) / N(\Lambda(a))\right. \\
S^{\phi} & =\lim _{a \rightarrow \infty} N(\Lambda(a))^{-1} S_{\varrho^{a}}(\Lambda(a)) .
\end{aligned}
$$

Proof. (i) and (ii) are consequences of Theorem 7.2 and the inequality

$$
P(\phi) \leqq \bar{s}\left(\varrho^{a}\right)-E\left(\varrho^{a} ; \phi\right)+\varepsilon, \quad \varepsilon \text { arbitrary small for large } a,
$$


which follows from Proposition 7.3 and the proof of Theorem 7.2. Let $\psi \in \overline{\mathscr{B}}_{R}^{f}$, then we have

$$
\begin{aligned}
P(\phi+\psi) & \geqq \bar{s}\left(\varrho^{a}\right)-E\left(\varrho^{a} ; \phi+\psi\right)=\bar{s}\left(\varrho^{a}\right)-E\left(\varrho^{a} ; \phi\right)-E\left(\varrho^{a} ; \psi\right) \\
& \geqq P(\phi)-E\left(\varrho^{a} ; \psi\right)-\varepsilon,
\end{aligned}
$$

and $\varepsilon$ tends to zero for any sequence $a_{v} \rightarrow \infty$ characterizing a point of accumulation, say, $E^{\phi}(\cdot)$ of $\left.\left\{E\left(Q^{a} ; \cdot\right)\right\} ; a \in \mathbb{Z}^{v}\right\}$. Due to $(46), E^{\phi}(\psi)$ is a tangent functional, and from (45), Eq. (42) follows with the corresponding point of accumulation $s^{\phi}$.

(iii) The uniqueness of $E^{\phi}$ and $s^{\phi}$ for $\phi \in T$ is trivial. The proof of (43) runs exactly as in the translation invariant case $[3,9]$ : The tangent functionals $\alpha_{\Lambda}^{\phi}$ to the graphs of $P_{A}(\cdot)$ are given by

$$
\alpha_{\Lambda}^{\phi}(\psi)=N(\Lambda)^{-1} \operatorname{Tr}_{\Lambda} e^{-H_{\Lambda}(\phi)} H_{\Lambda}(\psi) \cdot\left(\operatorname{Tr}_{\Lambda} e^{-H_{\Lambda}(\phi)}\right)^{-1}
$$

As is easily seen, any point of accumulation of $\left\{\alpha_{\Lambda}^{\phi} ; \Lambda \in H-R\right.$-sequence $\}$ gives a tangent functional to the graph of $P(\cdot)$. Due to the uniqueness, we have thus the first part of (43). Take the $H-R$-sequence $\{\Lambda(a), a \rightarrow \infty\}$, then the definition (33) of $\varrho^{a}$ yields the second equality in (43). Equation (44) is a trivial consequence of (35) and (43).

Q.E.D.

Finally let us add three remarks:

Remark 7.6. Let $\phi$ be a generalized RSI-potential characterized by $k$ sets $I_{j}$ and the probabilities $v_{j}\left(r_{j}, a\right), j=1, \ldots, k$. For a given $\Lambda(a)$ and its disjoint translates $\Lambda_{j}^{a}$, there are $k \cdot 2^{N(\Lambda(a))}$ different types of impurity distributions $d^{(r)}$, $r=1, \ldots, k \cdot 2^{N(\Lambda(a))}$. Let $d^{(r)}$ be realized for $\Lambda^{(r)}=\Lambda(a)+a^{(r)}$, where $\Lambda^{(r)}$ is one of the translates $\Lambda_{j}^{a}$. We put $H_{\Lambda(a)}^{(r)}=\tau_{-a^{(r)}} H_{\Lambda(a)+a^{(r)}}(\phi)$, and define $\varrho^{a, r}$ by the density matrices

$$
\begin{aligned}
\varrho_{\Lambda(a)}^{a, r}= & =e^{-H_{\Lambda(a)}^{(r)} / \operatorname{Tr}_{\Lambda(a)}} e^{-H_{\Lambda(a)}^{(r)}}, \\
\varrho_{\Lambda(a)+2 n a}^{a, r} & =\tau_{2 n a} \varrho_{\Lambda(a)}^{a, r}, \quad \varrho_{\cup}^{a, r} \Lambda_{j}^{a}=\prod^{\otimes} \varrho_{\Lambda_{j}^{a}}^{a, r} .
\end{aligned}
$$

Clearly, $\varrho^{a, r}$ is invariant under translations $\tau_{2 n a}$, and

$$
\bar{\varrho}^{a, r}(\cdot)=N(\Lambda(a))^{-1} \sum_{x \in \Lambda(a)} \varrho^{a, r}\left(\tau_{x} \cdot\right)
$$

is translation invariant. With $v(r ; a)=\prod_{j=1}^{k} v_{j}\left(r_{j} ; a\right)$, we define $\bar{\varrho}^{a}=\sum_{r} v(r, a) \bar{\varrho}^{a, r}$. Then we have $\varrho^{a} \in \mathscr{S}_{0}$ and $s\left(\varrho^{a}\right)=\bar{s}\left(\varrho^{a}\right)$. This is a consequence of $(\mathrm{R})$ and of the results of [2]. Unfortunately, one cannot approximate $P(\phi)$ with the help of $\bar{Q}^{a}$. For, in general,

$$
\lim _{\Lambda \rightarrow \infty} \bar{\varrho}^{a}\left(H_{\Lambda}(\phi) / N(\Lambda)\right) \geqq \lim _{\Lambda \rightarrow \infty} \varrho^{a}\left(H_{\Lambda}(\phi) / N(\Lambda)\right)=E\left(\varrho^{a} ; \phi\right)
$$

because $\varrho^{a}$ is better adapted to the fine structure of the potential $\phi$ in order for it to minimize the energy expectation value. An example will be found in the Appendix

Remark 7.7. In the translation invariant case, Theorem 7.2 has a converse [3]:

$$
s(\varrho)=\inf _{\phi \in \mathscr{B}_{1 / \xi}^{T}}\left(P(\phi)-\varrho\left(A_{\phi}\right)\right), \quad \varrho \in \mathscr{S}_{0} .
$$


One is tempted to conjecture that a similar equation holds for arbitrary $\varrho$ :

$$
\bar{s}(\varrho)=\inf _{\phi \in \overline{\mathscr{B}}_{R}^{1 / \xi}}(P(\phi)-E(\varrho ; \phi)), \quad \varrho \in \mathscr{S} .
$$

From Theorem 7.2 we readily derive

$$
\bar{S}(\varrho) \leqq \inf _{\phi \in \overline{\mathscr{B}}_{R}^{1 / \xi}}(P(\phi)-E(\varrho ; \phi)) .
$$

Let us assume that the $\varrho^{a}$ 's converge to some $\varrho^{e q}$ for which the supremum in Theorem 7.2 is attained. Then (50) would hold for $\varrho=\varrho^{e q}$. The proof of Theorem 7.2 shows that the structure of the $\varrho^{a}$ s - and hence that of $\varrho^{e q}$ - reflects the structure of $\phi$, especially its translational properties. Since $\phi$ has to be restricted to the class $\overline{\mathscr{B}}_{R}^{1 / \xi}$, we can at best expect (50) to hold for those $\varrho$ which are in some sense translation invariant in the mean, as are the generalized RSI-potentials.

Remark 7.8. If $\phi \in \overline{\mathscr{B}}_{R}^{f}$ with $f(\xi) \geqq e^{-\xi}$, then $\tau_{t}(\phi)$ exists, and one can look for KMS-states [11] with respect to $\tau_{t}(\phi)$. In the case of translation covariant potentials, it is known [3] that those states for which the supremum is obtained in (26) are KMS-states (with inverse temperature $\beta=1$ ) with respect to the time automorphism group defined by the potential. Because we do not have a $\varrho^{e q}$ in the general case, we can only ask the other way round: does a $\tau_{t}(\phi)$-KMS-state yield the supremum in Theorem 7.2? This has been an open question even in the invariant case until recently [12]. There seems to be a chance to carry over the proofs of [12] to the non-invariant case.

Acknowledgement. The problem treated in this paper arose from discussions with Prof. Winnink in Groningen whom I wish to thank. Furthermore, I am indebted to Prof. Borchers for his interest in the subject and for stimulating discussions during the phase of completion of the paper.

\section{Appendix: Potentials Exhibiting Partial Covariance}

Assume that $\phi$ is covariant under the subgroup $T_{a}=\left\{\tau_{n a}, a=\left(a^{1}, \ldots, a^{v}\right)\right.$, $\left.n=\left(n^{1}, \ldots, n^{v}\right), n^{\star}=0,1,2, \ldots\right\}$. We can apply the results for translation covariant potentials by taking the periodicity cells as elements of a new lattice $\tilde{\mathbb{Z}}^{v}:=\left\{Z_{0}+n a\right.$; $\left.n \in \mathbb{N}^{v}\right\}$, where $Z_{0}=\left\{x \in \mathbb{Z}^{v} ; 0 \leqq x^{\alpha}<a^{\alpha}, \alpha=1, \ldots, v\right\}$. To every subset

$$
\tilde{\Lambda}=\left\{Z_{0}+n_{\gamma} a ; \gamma \in \Gamma\right\} \subset \tilde{\mathbb{Z}}^{v}
$$

there corresponds a set $\Lambda \subset \mathbb{Z}^{v}: \Lambda=\bigcup_{\gamma \in \Gamma}\left(Z_{0}+n_{\gamma} a\right)$. We define $\tilde{\phi}$ by

$$
H_{\tilde{\Lambda}}(\tilde{\phi})=H_{\Lambda}(\phi)
$$

Notice that $\tilde{\phi}$ is uniquely defined if $H_{\tilde{\Lambda}}(\tilde{\phi})$ is given for all $\tilde{\Lambda} \subset \tilde{\mathbb{Z}}^{v}$. Clearly, $\tilde{\phi}$ is translation covariant under the group of $\tilde{\mathbb{Z}}^{v}$-lattice translations, hence the van Hove limit $\lim _{\tilde{\Lambda} \rightarrow \infty} P_{\tilde{\Lambda}}(\phi)$ exists, and, due to (A1), equals $N\left(Z_{0}\right) \lim _{\Lambda \rightarrow \infty} P_{\Lambda}(\phi)$. Here $\Lambda$ is meant to be restricted to unions of periodicity cells, but this restriction can be dropped according to Theorem 5.8 and Remark 5.9.

We can find a $\tilde{\mathbb{Z}}^{v}$-translation invariant equilibrium state $\varrho^{\tilde{\phi}}$ over the algebra $\tilde{\mathscr{A}}$ belonging to $\tilde{\mathbb{Z}}^{v}$ by defining states $\varrho^{b}$ in the way we defined $\varrho^{a, r}$ and $\varrho^{a, r}$ [just cancel the $r$ in (48) and (49), replace $\phi$ by $\tilde{\phi}$ and $\Lambda(a)$ by $\tilde{\Lambda}(b)]$; any point of 
accumulation of $\left\{\varrho^{b} ; \mathrm{b} \in \tilde{\mathbb{Z}}^{v}\right\}$ yields a $\varrho^{\tilde{\phi}}[2]$. Clearly, $\varrho^{\tilde{\phi}}$ can be considered as a state over the original algebra $\mathscr{A}$, moreover, it is an equilibrium state, i.e. we have $P(\phi)=s\left(\varrho^{\tilde{\phi}}\right)-\varrho^{\tilde{\phi}}\left(N\left(Z_{0}\right)^{-1} \sum_{x \in Z_{0}} A_{\phi}^{x}\right), s\left(\varrho^{\tilde{\phi}}\right)$ exists according to [2]; but, clearly, $\varrho^{\tilde{\phi}}$ is not $\mathbb{Z}^{v}$-translation invariant.

\section{References}

1. Ruelle, D.: Statistical mechanics. Amsterdam: W. A. Benjamin Inc. 1969

2. Robinson, D. W.: Commun. math. Phys. 7, 337 (1968)

3. Lanford, O.E., Robinson, D. W.: Commun. math. Phys. 9, 327 (1968)

4. Lima, R.: Commun. math. Phys. 24, 180 (1972)

5. Lieb, E.H., Ruskai, M. B.: Phys. Rev. Letters 30, 434 (1973)

6. Lieb, E.H., Ruskai, M. B.: J. Math. Phys. 14, 1938 (1973)

7. Roos, H.: Commun. math. Phys. 36, 263 (1974)

8. Lima, R., Naudts, J.: Spontaneous symmetry breakdown for quantum lattice systems. Marseille preprint 74/P. 604 (1974)

9. Gallavotti, G., Miracle-Sole, S: Commun. math. Phys. 5, 317 (1967)

10. Gallavotti, G., Miracle-Sole, S.: Commun. math. Phys. 7, 274 (1968)

11. See, for instance, Haag, R., Hugenholtz, N. M., Winnink, M.: Commun. math. Phys. 5, 215 (1967)

12. Araki, H.: Commun. math. Phys. 38, 1 (1974)

Communicated by G. Gallavotti

H. Roos

Institut für Theoretische Physik

Universität Göttingen

D-3400 Göttingen

Federal Republic of Germany

Note Added in Proof. There are three papers which should be cited, along with [8], in the context of averageable potentials:

13. Ruelle, D.: Ann. Phys. 69, 364 (1972)

14. Griffiths, R.B., Lebowitz, J.L.: J. Math. Phys. 9, 1284 (1968) and, as pointed out to me by the referee

15. Jacobs, K.: Math. Ann. 137, 125 (1959) 